\title{
NOTE ON A PROBLEM IN APPROXIMATION WITH AUXILIARY CONDITIONS*
}

\author{
BY DUNHAM JACKSON
}

Let $\rho(x)$ and $f(x)$ be two given functions of period $2 \pi$, the former bounded and measurable, with a positive lower bound, the latter, for simplicity, continuous. Among all trigonometric sums $T_{n}(x)$, of given order $n$, there is one and just one for which the value of the integral

$$
\int_{0}^{2 \pi} \rho(x)\left[f(x)-T_{n}(x)\right]^{2} d x
$$

is a minimum. If the weight function $\rho(x)$ is identically 1 ; it is a matter of familiar knowledge that the minimum is reached when $T_{n}(x)$ is the partial sum of the Fourier series for $f(x)$. A considerable amount of attention has been given recently to the problem of the convergence of the minimizing sum $T_{n}(x)$ toward $f(x)$, as $n$ becomes infinite, under the generalized conditions that result from the admission of an arbitrary weight function. $\dagger$

Let $x_{1}, \cdots, x_{N}$ be $N$ values of $x$ in the interval $0 \leqq x<2 \pi$. The problems of the preceding paragraph may be further varied by admitting to consideration only such sums $T_{n}(x)$ as satisfy the conditions

$$
T_{n}\left(x_{i}\right)=f\left(x_{i}\right), \quad(i=1,2, \cdots, N),
$$

and inquiring after the minimum of the integral (1) subject to these auxiliary conditions. It is understood that the given value of $n$ is large enough so that the conditions (2) can be

* Presented to the Society, April 3, 1926.

$\dagger$ Cf. e.g., D. Jackson, Note on the convergence of weighted trigonometric series, this Bulletin, vol. 29 (1923), pp. 259-263, where further bibliographical references will be found; also D. Jackson, A generalized problem in weighted approximation, Transactions OF THIs Society, vol. 26 (1924), pp. 133-154. 
fulfilled; for this it is sufficient that $n \geqq \frac{1}{2}(N-1)$. There is no essential novelty in the proof of the existence and uniqueness of the sum which yields the minimum. The notation $T_{n}(x)$ being restricted henceforth to this "approximating sum," it is the purpose of the following lines to discuss the convergence of $T_{n}(x)$ toward $f(x)$, as $n$ increases without limit. The question is not trivial, even if $\rho(x) \equiv 1$. It is quite distinct from the conventional problems of interpolation, inasmuch as $N$ is fixed, and does not increase with $n$.

For each value of $n\left(\geqq \frac{1}{2}(N-1)\right)$, let a continuous function $\varphi_{n}(x)$ be defined, having $\epsilon_{n}$ as an upper bound for its absolute value, and such that

$$
\varphi_{n}\left(x_{i}\right)=0, \quad(i=1,2, \cdots, N) .
$$

Let $\tau_{n}(x)$ be the approximating sum of the $n$th order for $\varphi_{n}(x)$; that is, the sum which minimizes the integral

$$
\int_{0}^{2 \pi} \rho(x)\left[\varphi_{n}(x)-\tau_{n}(x)\right]^{2} d x,
$$

subject to the conditions

$$
\tau_{n}\left(x_{i}\right)=0, \quad(i=1,2, \cdots, N) .
$$

Exactly as in the absence of the restrictions (3), (4), it may be shown* that

$$
\left|\varphi_{n}(x)-\tau_{n}(x)\right| \leqq k \epsilon_{n} \sqrt{n},
$$

where $k$ is independent of $n$ (expressible, in fact, in terms of the ratio of the upper and lower bounds of $\rho(x)$, and independent of anything else). The new conditions call for notice only to the extent of the observation that a trigonometric sum which vanishes identically comes within the requirements of (4). Furthermore, it is recognized at once that if $\varphi_{n}(x)$ is defined by the relation

$$
\varphi_{n}(x)=f(x)-t_{n}(x),
$$

where $t_{n}(x)$ is a trigonometric sum of the $n$th order taking on the same values as $f(x)$ at the points $x_{1}, x_{2}, \cdots, x_{N}$, then $\tau_{n}(x)$ and $T_{n}(x)$ are related by the identity $\dagger$

* D. Jackson, this Bulletin, loc. cit.

$\dagger$ Cf. this Bulletin, loc. cit., p. 261. 


$$
\tau_{n}(x)=T_{n}(x)-t_{n}(x),
$$

so that $f(x)-T_{n}(x)=\varphi_{n}(x)-\tau_{n}(x)$ indentically, and

$$
\left|f(x)-T_{n}(x)\right| \leqq k \epsilon_{n} \sqrt{n} .
$$

The formulation of sufficient conditions for convergence is reduced then to the determination of the order of magnitude of $\epsilon_{n}$, the measure of the accuracy with which $f(x)$ can be uniformly approached by trigonometric sums $t_{n}(x)$ such that

$$
t_{n}\left(x_{i}\right)=f\left(x_{i}\right), \quad(i=1,2, \cdots, N) .
$$

Let $y_{1}, y_{2} \cdots, y_{N}$ be any $N$ numbers subject to the conditions $\left|y_{i}\right| \leqq 1, i=1,2, \cdots, N$. If $N$ is even, let $x_{0}$ be a point in $(0,2 \pi)$ distinct from $x_{1}, x_{2}, \cdots, x_{N}$, and let $y_{0}=1$. Let $t(x)$ be the trigonometric sum of order $\frac{1}{2}(N-1)$ or $\frac{1}{2} N$, according as $N$ is odd or even, which takes on the values $\left[y_{0}\right], y_{1}, \cdots, y_{N}$ at the points $\left[x_{0}\right], x_{1}, \cdots, x_{N}$. Let $g$ be the maximum of $|t(x)|$. This $g$ is a continuous function* of $y_{1}, y_{2}, \cdots, y_{N}$, and has a maximum $G$, as the $y$ 's range over all admissible values. If 1 is replaced by $\eta$ as upper bound for the absolute values of the $y$ 's, the greatest possible absolute value of the corresponding $t(x)$ is $G \eta$.

Now suppose it is known that for each $n \geqq \frac{1}{2} N$ there is a trigonometric sum $\bar{t}_{n}(x)$, of the $n$th order, satisfying everywhere the relation

$$
\left|f(x)-\bar{t}_{n}(x)\right| \leqq \eta_{n},
$$

but not further specially restricted at the points $x_{1}, \cdots, x_{N}$. Let

$$
y_{i}=f\left(x_{i}\right)-\bar{t}_{n}\left(x_{i}\right), \quad(i=1,2, \cdots, N),
$$

and let $t(x)$ be determined as above, for this set of $y$ 's. Then $|t(x)| \leqq G \eta_{n}$, where $G$ is independent of $n$ (being dependent only on $\left.x_{1}, \cdots, x_{N}\right)$. The determination of

\footnotetext{
* Explicitly, as is well known,

$t(x)=\sum_{i=1}^{N} y_{i} \frac{\sin \frac{1}{2}\left(x-x_{1}\right) \cdots \sin \frac{1}{2}\left(x-x_{i-1}\right) \sin \frac{1}{2}\left(x-x_{i+1}\right) \cdots \sin \frac{1}{2}\left(x-x_{N}\right)}{\sin \frac{1}{2}\left(x_{i-1} x_{1}\right) \cdots \sin \frac{1}{2}\left(x_{i-x_{i-1}}\right) \sin \frac{1}{2}\left(x_{i}-x_{i+1}\right) \cdots \sin \frac{1}{2}\left(x_{i}-x_{N}\right)}$
}

when $N$ is odd, the initial index 1 being replaced by 0 when $N$ is even. 
$t(x)$ is different for different values of $n$, but each $t(x)$ is itself a trigonometric sum of order $\frac{1}{2} N$ at most. The identity

$$
t_{n}(x)=\bar{t}_{n}(x)+t(x)
$$

defines a sum of the $n$th order such that the conditions (5) are fulfilled, and such that

$$
\left|f(x)-t_{n}(x)\right| \leqq(1+G) \eta_{n} .
$$

As the factor $1+G$ is independent of $n$, this means that the order of the attainable approximation is not affected by the imposition of the restrictions (5).

In particular, if $\omega(\delta)$ is the maximum of $\left|f\left(x^{\prime}\right)-f\left(x^{\prime \prime}\right)\right|$ for $\left|x^{\prime}-x^{\prime \prime}\right| \leqq \delta$, and if $\lim _{\delta \rightarrow 0} \omega(\delta) / \sqrt{\delta}=0$, sums $\bar{t}_{n}(x)$ will exist* such that $\lim _{n \rightarrow \infty} \eta_{n} \sqrt{n}=0$, and there will consequently be sums $t_{n}(x)$ such that $\lim _{n \rightarrow \infty} \epsilon_{n} \sqrt{n}=0$. We may state the result as a theorem, identical in form with the one found when the auxiliary conditions (2) are omitted.

THEOREM. The sum $T_{n}(x)$ will converge uniformly to the value $f(x)$ for $n \rightarrow \infty$, provided that

$$
\lim _{\delta \rightarrow 0} \omega(\delta) / \sqrt{\delta}=0 .
$$

It is readily seen that essentially the same treatment can be carried through if $\left[f(x)-T_{n}(x)\right]^{2}$ in (1) is replaced by $\left|f(x)-T_{n}(x)\right|^{m}$, for any value of $m>1$; the condition for convergence is that $\lim _{\delta \rightarrow 0} \omega(\delta) / \delta^{1 / m}=0$. The discussion can be further extended in various ways that need not be elaborated here.

The University of Minnesota

* Cf. this Bulletin, loc. cit., p. 261. 